\title{
Zanamivir speeds symptom relief in influenza
}

\author{
Monto AS, Fleming DM, Henry D, et al. Efficacy and safety of the neuraminidase inhibitor zanamivir in the treatment of \\ influenza A and B virus infections. J Infect Dis 1999 Aug;180:254-61.
}

\section{QUESTION: In patients with symptoms of influenza, what is the clinical efficacy and safety of zanamivir?}

Design

Randomised \{allocation concealed*i†, blinded (clinicians and patients),* placebo controlled trial with 21 day follow up.

Setting

Clinical centres in North America and Europe.

\section{Patients}

1256 patients (mean age $35 \mathrm{y}, 66 \%$ women) who presented with symptoms of influenza of $\leqslant 48$ hours of duration during 1 influenza season: fever and $\geqslant 2$ of myalgia, headache, cough, or sore throat. Exclusion criteria were unstable chronic illness, receipt of other antiviral agents in the previous 7 days, inability to use inhaler devices satisfactorily, known or suspected hypersensitivity to study medication, pregnancy, lactation, or potential for pregnancy. 1182 patients (94\%) completed the study.

\section{Intervention}

Patients were allocated to 5 days of treatment with zanamivir, $10 \mathrm{mg}$ by oral inhalation and $6.4 \mathrm{mg}$ by nasal spray 2 times/day $(\mathrm{n}=419)$; zanamivir (same dosages and administration routes) 4 times/day $(n=415)$; or placebo $(\mathrm{n}=422)$.

\section{Main outcome measures}

Time to alleviation of clinical symptoms, which had to be maintained for $\geqslant 24$ hours. Secondary outcomes were mean symptom score, sleep disturbance, time to return to normal activities, and use of acetaminophen and dextromethorphan to relieve symptoms.

\section{Main results}

Analysis was by intention to treat. Both regimens of zanamivir reduced the time to alleviation of clinical symptoms by 1 day less than placebo $(6 v 7 \mathrm{~d}, \mathrm{p} \leqslant 0.014)$. The 2 drug groups did not differ $(p=0.77)$. In patients who entered the study within 30 hours of the onset of symptoms, symptom duration was reduced by 1 day with zanamivir 2 times $/ \mathrm{d}(\mathrm{p}=0.015)$ and by 1.5 days with zanamivir 4 times $/ d(p=0.001)$. Patients who were febrile at study entry $\left(\geqslant 37.8^{\circ} \mathrm{C}\right)$ had reduced symptom duration by 1.5 days with zanamivir 2 times/day $(\mathrm{p}=0.049)$ and by 2 days with zanamivir 4 times/day $(\mathrm{p}=0.032)$. In patients considered at high risk for developing complications (age $\geqslant 65$ y or cardiovascular, respiratory, endocrine, or metabolic conditions), zanamivir 4 times/day reduced time to symptom relief by 2.8 days $(\mathrm{p}=0.042)$, and both regimens were more effective than placebo in high risk patients who had positive findings for influenza $(\mathrm{p} \leqslant 0.016)$. Zanamivir was superior to placebo in all secondary clinical outcomes. The groups did not differ for adverse events.

\section{Conclusions}

In patients with symptoms of influenza, zanamivir given 2 or 4 times per day decreased the time to alleviation of symptoms by 1 to 1.5 days. Patients who presented early after onset of symptoms with fever $\geqslant 37.8^{\circ} \mathrm{C}$ or who were at high risk for complications had the most benefit.

*See glossary.

$\dagger$ Information provided by author.

\section{COMMENTARY}

Influenza remains a major cause of morbidity and mortality each year. Although vaccination is the primary method for preventing influenza, antiviral agents play an important complementary role for preventing and treating the disease.

In 1999, zanamivir and oseltamivir were approved for the treatment of influenza A and B. ${ }^{1}$ Before 1999, amantadine and rimantadine were the only antiviral agents available and were active only against influenza A viruses. Their use has been limited by side effects (especially related to the central nervous system) and the rapid emergence of resistant viruses. The neuraminidase inhibitors appear to be much less likely to induce resistance. They are also generally well tolerated, as shown in these studies by Monto and Hayden and their colleagues, although zanamivir has been reported to induce bronchospasm and oseltamivir has been associated with gastrointestinal symptoms.'

Zanamivir and oseltamivir are effective for the treatment of influenza A or B among adults when started within 2 days of onset of symptoms. Monto and colleagues administered zanamivir by nasal spray and in its currently available form of orally inhaled powder.

Duration of influenza was reduced by about 1 day, with greater benefits for patients with more severe (eg, febrile) illness and when started within 30 hours of onset of symptoms. 2 other trials reported similar findings: zanamivir relieved symptoms 1 to 1.5 days earlier. ${ }^{2}$ Oral oseltamivir also reduces the duration of influenza illness among healthy adults by about 30 hours when started within 1.5 days of the onset of symptoms.

Most of the available data on the efficacy of the neuraminidase inhibitors are from healthy adults $<65$ years of age. $13 \%$ of participants in the study by Monto and colleagues were high risk (age $\geqslant 65 \mathrm{y}$ or with stable chronic medical conditions). The study findings suggested that zanamivir was also effective in this subgroup, but the sample size was too small to detect a statistically significant benefit. More data are needed to define clearly the role of the neuraminidase inhibitors in high-risk groups.

Although not approved for prophylaxis, zanamivir and oseltamivir have also been shown to prevent influenza in healthy adults. Hayden and colleagues found oseltamivir given for 6 weeks was $74 \%$ effective in preventing influenza. Furthermore, a trial of a 4 week course of zanamivir in 1107 adults showed a $67 \%$ efficacy in preventing influenza and an $84 \%$ efficacy in preventing febrile influenza. ${ }^{5}$ Of note, Hayden and colleagues reported that 7 participants developed influenza continued on next page 